\title{
NUCLEAR PHYSICS Advancing on Several Fronts
}

\section{EUROBALL Agreement Signed}

The collaboration agreement for the ambulatory $\gamma$-ray nuclear physics detector EUROBALL was finalised in Milan on 1 July. The so-called Legnaro phase envisages that EUROBALL will be operated at the INFN's ALPI accelerator in Legnaro for 14-16 months starting at the end of 1996 (construction of a new experimental hall has started). It will then be moved to the VIVITRON accelerator at the Centre de Recherche Nucléaire (CRN), Srasbourg. The detector configuration will be a full $4 \pi$-array with coaxial detectors from EUROGAM I, clover detectors from EUROGAM II and 16 new cluster detectors that have been ordered. There is also a development phase for new detectors. P. Twin from Liverpool who chaired the collaboration's committee feels that EUROBALL is a natural evolution to larger facilities (six countries are involved).

\section{VIVITRON Operating}

B. Heusch, the project leader of VIVITRON speaking at the European Particle Accelerator Conference (London; 27 June - 1 July) reported that the first physics experiment with a beam was expected the same week. EUROGAM is now installed at the VIVITRON having received its first beam in January after moving from Daresbury. Only 3 of the world's approximately 20 tandem accelerators operate above $17 \mathrm{MeV}$, and the VIVITRON is the last to be available for high-precision work now that the others are to be shut down (Yale, USA) or used for radioactive beams (Oak Ridge, USA). Following extensive work on the horizontal insulation structures responsible for problems with beam stability, the CRN is confident that the VIVITRON can operate routinely at $15-20 \mathrm{MeV}$ (more than 24 hours have been achieved between spark discharges). The plan is to operate for 12 months to see how the machine settles down before committing to a possible upgrading to $30 \mathrm{MeV}$ involving further changes to the insulators. A second ambulatory detector (DEMON) will arrive in Strasbourg at the end of the year (it will soon move from Louvainla-Neuve to GANIL in Caen).

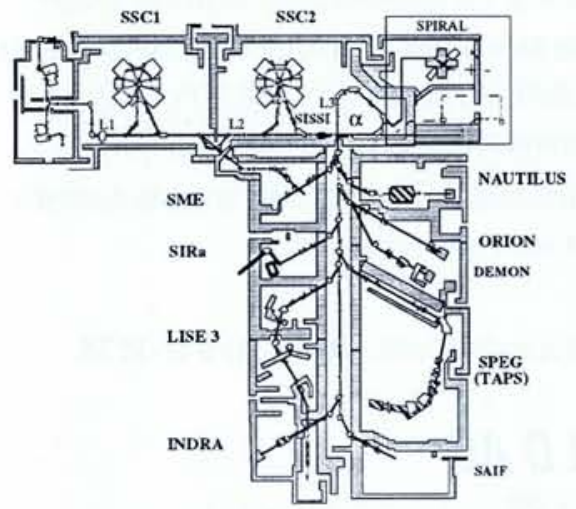

A plan of the GANIL laboratory showing the new SPIRAL radioactive-beam facility that is under construction (completion is planned for mid-1997 with beams available in 1998). The primary beam will be led from the existing SSC2 synchrotron to an ion source.
$A$ view along LISA showing the four superconducting RF cavities. Powered by $15 \mathrm{~kW}$ continuous wave klystrons, they operate at $5 \mathrm{MV} / \mathrm{m}$.

The French-Belgian gamma-ray detector DEMON that will move to the VIVITRON accelerator in Strasbourg this year.

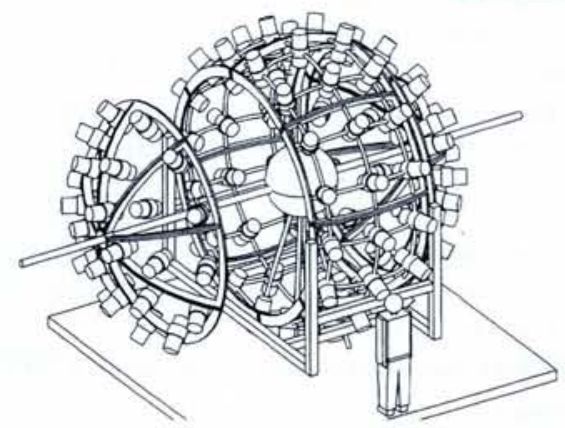

Radioactive-Beam Facility Approved

GANIL received approval last November for SPIRAL, Europe's first major, national radioactive-beam facility (a much smaller facility in Louvain-la-Neuve has operated since 1989). Radioactive ions formed by the interaction of the recently upgraded, highintensity GANIL primary beam (Ne through to Ar) with a thick target will be collected and ionized, separated and accelerated (the socalled ISOL method) and re-injected into an analyzing spectrometer upstream of the experimental areas. The main new item of the $100 \mathrm{MFF}$ project is a $2-25 \mathrm{MeV}$ per nucleon compact cyclotron post-accelerator with its associated low-energy and highenergy beam-lines installed in an existing experimental area.

\section{Italy's Superconducting Accelerators}

Commissioning of the ALPI superconducting accelerator at the INFN's national laboratory in Legnaro, which started in January, has progressed to the point that the first beam $\left({ }^{58} \mathrm{Ni}^{12+}\right.$ at $345 \mathrm{MeV}$, equivalent to a $27.5 \mathrm{MeV}$ tandem voltage) was extracted to the GASP target area with the mediumbeta central part of the accelerator in operation (GASP is a new $4 \pi$ high-resolution gamma-ray spectrometer which is coupled to an existing recoil-mass spectrometer). The experiment ran for 2 days before the breakdown of a cryogen liquifier. A preliminary analysis was made of the $\gamma-\gamma$ coincidences for a ${ }^{208} \mathrm{~Pb}$ target and up to 14

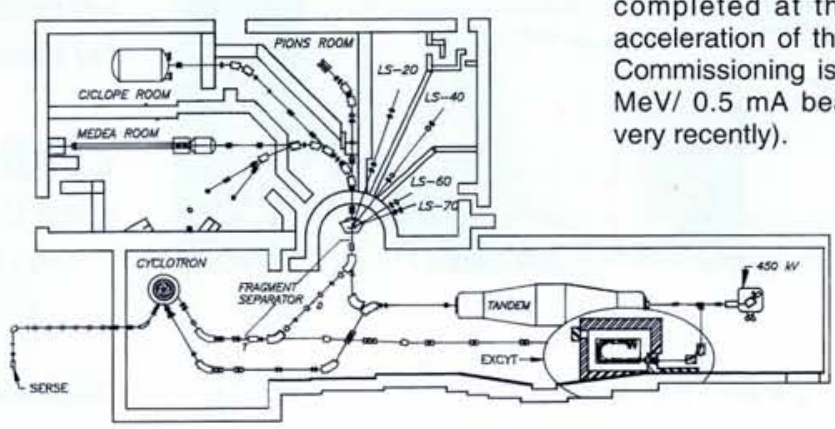

neutron exchanges were detected. Achieving the energy is the relatively easy part; the challenge is to raise the intensity. The plan is to proceed with the installation of the lowbeta part of the machine and bring the energy to $40 \mathrm{MeV}$ by 1997.

Italy has two other accelerator-based projects that are according to L. Maiani, the President of the INFN, can be seen "in a way" as launching Italy into the exploitation of superconducting accelerator technology. One is the superconducting cyclotron at the INFN, Laboratori Nazionale del Sud, Catania, where a $\mathrm{Ni}$ beam was successfully transported from a $15 \mathrm{MeV}$ tandem accelerator to the stripper foil in June. The first acceleration test was interrupted by the failure of a liquifier. The beam extraction system is now being installed and the plan is to extract the first beam in the autumn. The cyclotron was built in Milan and was moved to Catania in 1990.

The third project is the $25 \mathrm{MeV}$ superconducting radio-frequency electron linac LISA at the Laboratori Nazionali di Frascati, which is now a test machine for problems relating to a superconducting version of a possible next-generation linear collider (the very high integrated charge in the machine's $2 \mathrm{~mA}$ macropulses makes it very useful for testing beam diagnostic elements for colliding beams). Started in 1989 as part of a special national project for a general-purpose collider, it is used to test instrumentation and techniques as part of the TESLA Test Facility project at DESY. By incorporating an undulator and an optical cavity, LISA was designed to act as a beam generator for a fee-electron laser (FEL) in the infrared of the type that is attracting considerable interest world-wide. Proposed as an autonomous experiment in collaboration with ENEA, the agency that carries out research into new technology, energy and the environment, the FEL programme was cancelled in 1992. S. Tazzari, the project leader, writes to say that construction of LISA was essentially completed at the end of 1993 with the acceleration of the first beam in December Commissioning is now well advanced (a 20 $\mathrm{MeV} / 0.5 \mathrm{~mA}$ beam has been accelerated The plan of the nuclear physics facility at the INFN's laboratory in Catania. The superconducting cyclotron is located to the left of the existing tandem accelerator. 